Internat. J. Math. \& Math. Sci.

Vol. 1 (1978) 203-208

\title{
A NOTE ON STRICTLY CYCLIC SHIFTS ON $l_{p}$
}

\author{
GERD H. FRICKE
}

Department of Mathematics

Wright State University

Dayton, Ohio 45431

(Received June 28, 1977)

ABSTRACT. In this paper the author shows that a well known sufficient condition for strict cyclicity of a weighted shift on $l_{p}$ is not a necessary condition for any $\mathrm{p}$ with $1<\mathrm{p}<\infty$.

1. INTRODUCTION.

For $1 \leq \mathrm{p}<\infty$ let $\ell_{\mathrm{p}}$ be the Banach space of absolutely p-summable sequences of complex numbers. Let $s_{\alpha}$ denote the weighted shift on $l_{p}$ with weight sequence $\alpha=\left\{\alpha_{n}\right\}_{1}^{\infty}$ defined by $s_{\alpha}\left[\sum_{n=0}^{\infty} x_{n} e_{n}\right]={ }_{n=1}^{\infty} \alpha_{n} x_{n-1} e_{n}$. Let $\beta_{0}=1$ and $\beta_{\mathrm{n}}=\alpha_{1} \alpha_{2} \ldots \alpha_{\mathrm{n}}$ for all $\mathrm{n} \geq 1$. (For more detail we refer the reader to [2] and [3]).

Mary Embry [1] showed that for $\mathrm{p}=1$ the weighted shift $\mathrm{S}_{\alpha}$ is strictly cyclic if and only if

$$
\sup _{n, m}\left|\frac{\beta_{n+m}}{\beta_{n} \beta_{m}}\right|<\infty .
$$


Edward Kerlin and Alan Lambert [2] considered the natural extension to (1.1) for the case $1<p<\infty$ with $\frac{1}{p}+\frac{1}{q}=1$,

$$
\sup _{n} \sum_{m=0}^{n}\left|\frac{\beta_{n}}{\beta_{m} \beta_{n-m}}\right|^{q}<\infty
$$

and showed (1.2) implies $s_{\alpha}$ is strictly cyclic on $\ell_{p}$. They also proved that (1.2) is necessary if the weight sequence $\alpha$ is eventually decreasing.

This strongly suggested that (1.2) is a necessary and sufficient condition for strict cyclicity. However, we will show in this paper that (1.2) is not a necessary condition for $S_{\alpha}$ to be strictly cyclic for any $\mathrm{p}$ with $1<\mathrm{p}<\infty$.

2. PROOF.

To preserve the clarity of the proof we will consider the cases $1<p \leq 2$ and $2<\mathrm{p}<\infty$ separately.

(a) Let $1<p \leq 2$ and let $q$ be such that $\frac{1}{p}+\frac{1}{q}=1$. Let $\left\{n_{k}\right\}_{1}^{\infty}$ be a sequence of rapidly increasing positive integers, e.g., choose $n_{1}=10$ and $\mathrm{n}_{\mathrm{k}}=\left(10 \mathrm{n}_{\mathrm{k}-1}\right)^{10 \mathrm{q} \mathrm{n}_{\mathrm{k}-1}}$ for $\mathrm{k}>1$.

We now define the weight sequence $\alpha=\left\{\alpha_{i}\right\}_{1}^{\infty}$ by $\alpha_{1}=\alpha_{2}=\ldots=\alpha_{n_{1}}=1$ and for $k>1$

$$
\alpha_{i}= \begin{cases}n_{k}^{-1} & \text { if } n_{k-1}<i \leq n_{k}-n_{k-1} \\ n_{k-1}^{-1} & \text { if } n_{k}-n_{k-1}<i \leq n_{k} .\end{cases}
$$

Clearly, $\alpha_{n_{k-1}+1}=\alpha_{n_{k-1}+2}=\ldots=\alpha_{n_{k}-n_{k-1}}$ and $\alpha_{n_{k}-\ell+1} \leq \alpha_{\ell}$ for $1 \leq \ell \leq \frac{n_{k}}{2}$.

Thus, for $0<m<n_{k}$

$$
\frac{\beta_{n_{k}}}{\beta_{m}^{\beta} n_{k}-m}=\frac{\alpha_{n_{k}-m+1} \alpha_{n_{k}-m+2} \cdots \alpha_{n_{k}}}{\alpha_{1} \alpha_{2} \ldots \alpha_{m}^{\alpha}} \geq \frac{\alpha_{n_{k}-n_{k-1}+1} \ldots \alpha_{n_{k}}}{\alpha_{1} \alpha_{2} \ldots \alpha_{n_{k-1}}} \geq\left(\alpha_{n_{k}}\right)^{n_{k-1}}
$$


Therefore,

$$
\sum_{m=1}^{n_{k}-1}\left|\frac{\beta_{n_{k}}}{\beta_{m} \beta_{n_{k}-m}}\right|^{q} \geq n_{k}\left(\alpha_{n_{k}}\right)^{q n_{k-1}}=n_{k} n_{k-1}^{-q n_{k-1}} \rightarrow \infty \text { as } k \rightarrow \infty .
$$

Hence,

$$
\sup _{n} \sum_{m=0}^{n}\left|\frac{\beta_{n}}{\beta_{m} \beta_{n-m}}\right|^{q}=\infty .
$$

We now show that $S_{\alpha}$ is strictly cyclic. It is known that $s_{\alpha}[2]$ is strictly cyclic if and only if

$$
\sum_{n=0}^{\infty}\left|\sum_{m=0}^{n} \frac{\beta_{n}}{\beta_{m}^{\beta} \beta_{n-m}} x_{m} y_{n-m}\right|^{p}<\infty \text { for all } x, y \in \ell_{p} .
$$

Obviously, for $0<\mathrm{m}<\mathrm{n}$

$$
\frac{\beta_{n}}{\beta_{m} \beta_{n-m}}=\frac{\alpha_{n-m+1} \cdots \alpha_{n}}{\alpha_{1} \alpha_{2} \cdots \alpha_{m}} \leq \alpha_{n}
$$

and $\frac{\beta_{n}}{\beta_{m} \beta_{n-m}}=1$ for $m=0$ or $m=n$.

Thus,

$$
\begin{aligned}
\left.\left.\sum_{n=0}^{\infty}\right|_{m=0} ^{n} \frac{\beta_{n}}{\beta_{m} \beta_{n-m}} x_{m} y_{n-m}\right|^{p} & \leq \sum_{n=0}^{\infty}\left\{\left|x_{0} y_{n}\right|+\left|y_{0} x_{n}\right|+\sum_{m=1}^{n-1} \alpha_{n}\left|x_{m} y_{n-m}\right|\right\}^{p} \\
& \leq \sum_{n=0}^{\infty}\left\{\left|x_{0} y_{n}\right|+\left|y_{0} x_{n}\right|+\alpha_{n}\|x\|\left\|_{2}\right\| y||{ }_{2}\right\}^{p}<\infty \\
& \text { since } \alpha, x, y \varepsilon \ell_{p} .
\end{aligned}
$$

Hence (1.3) holds and $S_{\alpha}$ is strictly cyclic.

(b) Let $2<p<\infty$ and let $\frac{1}{p}+\frac{1}{q}=1$. Let $\left\{n_{k}\right\}_{1}^{\infty}$ be a sequence of rapidly increasing integers, e.g., choose $n_{1}=10$ and $n_{k}$ such that

$$
\sum_{n=1}^{n_{k}} \frac{1}{n} \geq\left(10 n_{k-1}\right)^{10 n_{k-1}} \text { for } k>1
$$


Define $\left\{d_{i}\right\}_{1}^{\infty}$ such that $\prod_{i=1}^{n} d_{i}=n^{-\frac{1}{q}}$ for $n=1,2, \cdots$ and define $\left\{s_{k}\right\}_{1}^{\infty}$ by $s_{1}=10$ and $s_{k}=2 s_{k-1}+2 n_{k}$ for $k>1$.

We now define the weight sequence $\alpha=\left\{\alpha_{1}\right\}_{1}^{\infty}$ by $\alpha_{1}=\ldots=\alpha_{s_{1}}=1$ and for $k>1$,

$$
\alpha_{1}=\left\{\begin{array}{lll}
n_{k}^{-2} & \text { if } & s_{k-1}<i \leq s_{k-1}+n_{k} \\
n_{k}^{-2} d_{s_{k}-s_{k-1}-i+1} & \text { if } & s_{k-1}+n_{k}<i \leq s_{k}-s_{k-1} \\
n_{k-1}^{-2} & \text { if } & s_{k}-s_{k-1}<1 \leq s_{k} .
\end{array}\right.
$$

Now, for $s_{k-1}<m<\frac{s_{k}}{2}$,

$$
\begin{aligned}
& \frac{{ }^{\beta} s_{k}}{\beta_{m}^{\beta} s_{k}-m}=\frac{\alpha_{s_{k}-m+1} \cdots \alpha_{s_{k}}}{\alpha_{1} \alpha_{2} \cdots \alpha_{m}} \geq \frac{\alpha_{s_{k}-m+1} \cdots \alpha_{s_{k}-s_{k-1}}}{\alpha_{s_{k-1}+1} \cdots \alpha_{m}} n_{k-1} s_{k-1} \geq n_{k-1}^{-2 s_{k-1}} \prod_{i=1}^{m-s_{k-1}} d_{1} \\
& =n_{k-1}^{-2 s_{k-1}\left(m-s_{k-1}\right)^{-\frac{1}{q}}}
\end{aligned}
$$

Thus,

$$
\sum_{m=0}^{s_{k}}\left|\frac{\beta_{s_{k}}}{\beta_{m} s_{k}-m}\right|^{q} \geq n_{k-1}-2 q s_{k-1} \sum_{i=1}^{n_{k}} i^{-1} \geq n_{k-1}-10 n_{k-1} \sum_{i=1}^{n_{k}} i^{-1} \rightarrow \infty \text { as } k \rightarrow \infty .
$$

Hence,

$$
\sup _{n} \sum_{m=0}^{n}\left|\frac{\beta_{n}}{\beta_{m} \beta_{n-m}}\right|^{q}=\infty \text {. }
$$

We now show that

$$
\sum_{n=0}^{\infty}\left|\sum_{m=0}^{n} \frac{\beta_{n}}{\beta_{m} \beta_{n-m}} x_{m} y_{n-m}\right|^{p}<\infty \text { for all } x, y \in \ell_{p} \text {. }
$$

If $s_{k-1}<n<s_{k}-s_{k-1}$ and $0<m<n$ then, $\quad \frac{\beta_{n}}{\beta_{m} \beta_{n-m}} \leq n_{k}^{-2}$. 
Let $\mathrm{h}=\min \{\mathrm{m}, \mathrm{n}-\mathrm{m}\}$ then, for $\mathrm{s}_{\mathrm{k}}-\mathrm{s}_{\mathrm{k}-1} \leq \mathrm{n} \leq \mathrm{s}_{\mathrm{k}}$ and $0<\mathrm{m}<\mathrm{n}$,

$$
\frac{\beta_{n}}{\beta_{m}^{\beta} n-m} \leq n_{k-1}^{-2} h^{-\frac{1}{q}} \text {. }
$$

Thus,

$$
\sum_{k=2}^{\infty} \underset{n=s_{k-1}+1}{s_{k}-s_{k-1}-1}\left|\sum_{m=1}^{n-1} \frac{\beta_{n}}{\beta_{m} \beta_{n-m}} x_{m} y_{n-m}\right|^{p} \leq \sum_{k=2}^{\infty} \sum_{n=s_{k-1}+1}^{s_{k}-s_{k-1}-1}\left(\left.n_{k}^{-2} \sum_{m=1}^{n-1}\left|x_{m} y_{n-m}\right|\right|^{p}<\infty .\right.
$$

Let $\delta=\frac{p}{p-2}$ then $\delta>q$ and $\frac{1}{\delta}+\frac{2}{p}=1$. Let $M=\left(\sum_{m=1}^{\infty} 2 m-\frac{\delta}{q}\right)^{\frac{1}{\delta}}\left[\|x\|_{p}^{p}+\|y\|_{p}^{p}\right)^{\frac{2}{p}}<\infty$. Then,

$$
\begin{aligned}
& \sum_{k=2}^{\infty} \underset{n=s_{k}-s_{k-1}}{s_{k}}\left|\sum_{m=1}^{n-1} \frac{\beta_{n}}{\beta_{m} \beta_{n-m}} x_{m} y_{n-m}\right|^{p} \\
& \leq \sum_{k=2}^{\infty} \underset{n=s_{k}-s_{k-1}}{s_{k}}\left(\sum_{m=1}^{\infty} n_{k-1}^{-2} h^{-\frac{1}{q}}\left|x_{m} y_{n-m}\right|\right)^{p} \text {, where } h=\min \{m, n-m\} \\
& \leq \sum_{k=2}^{\infty} \underset{n=s_{k}-s_{k-1}}{s_{k}} n_{k-1}^{-2 p} M^{p}<\infty .
\end{aligned}
$$

Combining (1.4) and (1.5) we obtain that (1.3) is satisfied. QED

\section{References}

1. Embry, Mary. Strictly Cyclic Operator Algebras on a Banach Space, to appear in Pac. J. Math. 2. Kerlin, Edward and Alan Lambert. Strictly Cyclic Shifts on $\ell_{p}$, Acta Sci.
Math. 35 (1973) 87-94.

3. Lambert, Alan. Strictly Cyclic Weighted Shifts, Proc. Amer. Math. Soc. 29 (1971) 331-336. 
KEY WORDS AND PHRASES. Strictly cyclic shifts, absolutely p-summable sequence, $\ell_{p}$-space, cyclic operator algebras.

AMS(MOS) SUBJECT CLASSIFICATION (1970) CODES. $40 H 05$. 


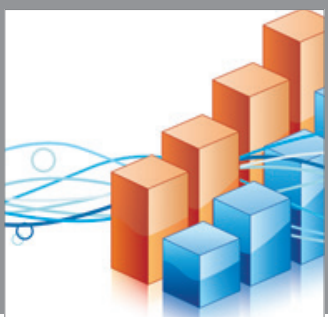

Advances in

Operations Research

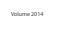

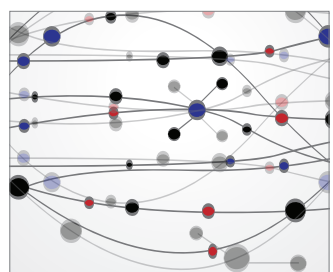

\section{The Scientific} World Journal
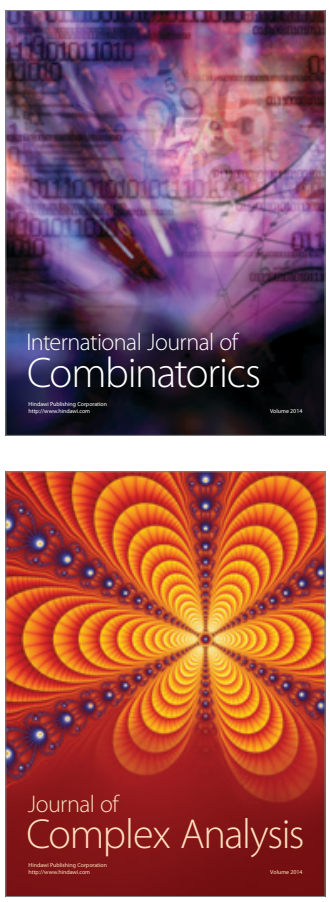

International Journal of

Mathematics and

Mathematical

Sciences
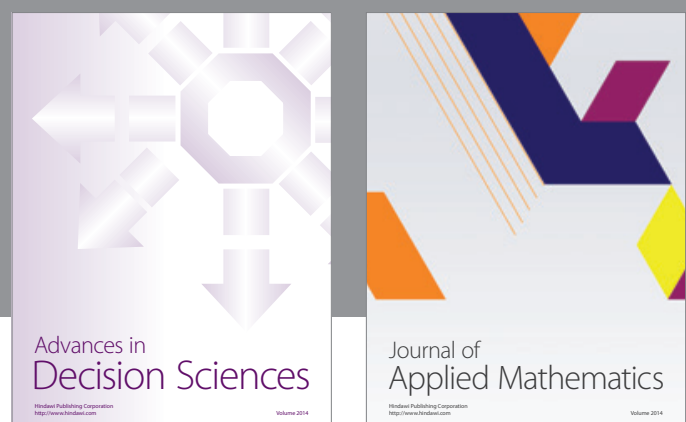

Journal of

Applied Mathematics
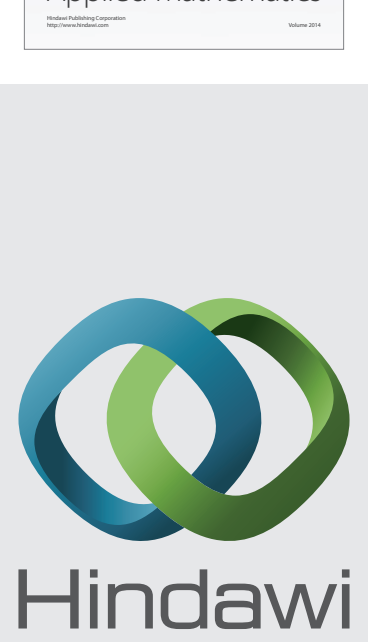

Submit your manuscripts at http://www.hindawi.com
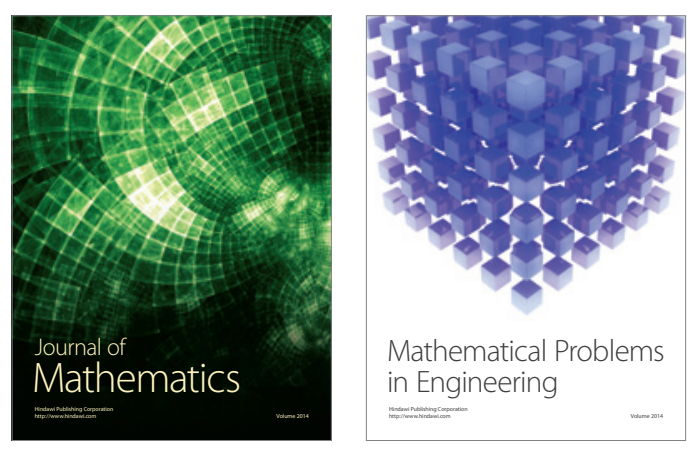

Mathematical Problems in Engineering
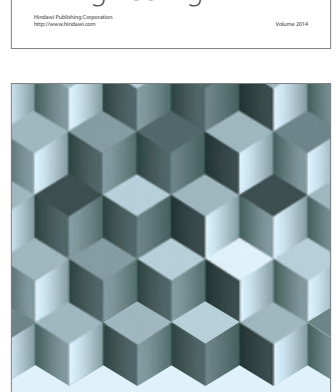

Journal of

Function Spaces
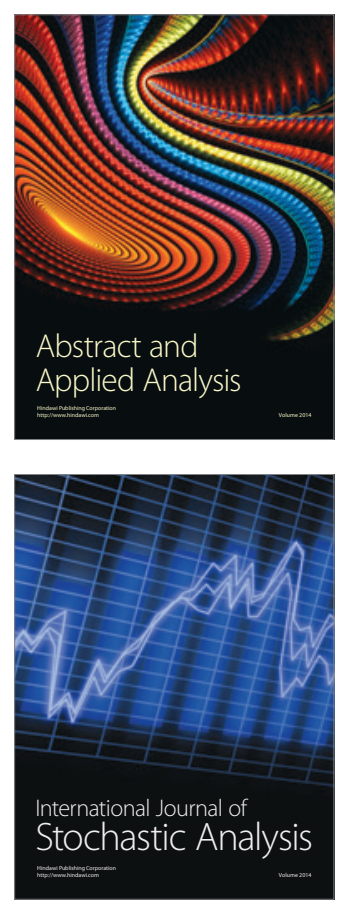

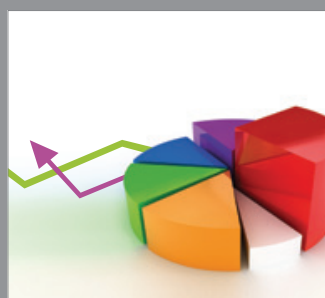

ournal of

Probability and Statistics

Promensencen
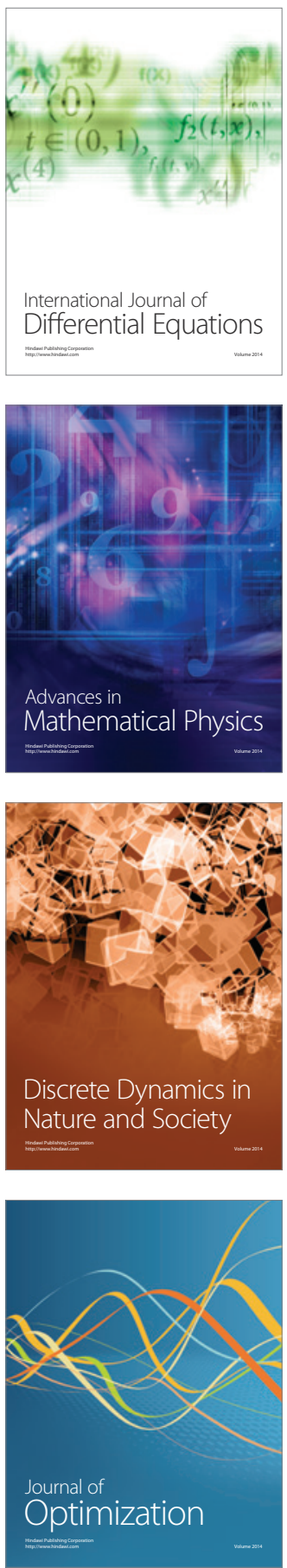